\title{
CONSTRAINING LANDSCAPE HISTORY AND GLACIAL EROSIVITY USING PAIRED COSMOGENIC NUCLIDES IN UPERNAVIK, NORTHWEST GREENLAND Online Data Repository
}

\author{
Lee B. Corbett* ${ }^{1,2}$, Paul R. Bierman ${ }^{1}$, Joseph A. Graly ${ }^{1,3}$, \\ Thomas A. Neumann ${ }^{1,4}$, and Dylan H. Rood ${ }^{5,6}$ \\ * Contact Author: Ashley.Corbett.GR@dartmouth.edu \\ ${ }^{1}$ Department of Geology, University of Vermont, Burlington, VT 05405 \\ ${ }^{2}$ Current affiliation: Department of Earth Sciences, Dartmouth College, Hanover, NH 03755 \\ ${ }^{3}$ Current affiliation: Department of Geology and Geophysics, University of Wyoming, Laramie, WY 82071 \\ ${ }^{4}$ Current affiliation: NASA Goddard Space Flight Center, Cryospheric Sciences Branch, Greenbelt, MD 20770 \\ ${ }^{5}$ Scottish Universities Environmental Research Centre (SUERC), East Kilbride G75 0QF, UK \\ ${ }^{6}$ Earth Research Institute, University of California, Santa Barbara, CA 93106 USA
}

\section{Isotopic analysis}

For Be analysis, samples were processed in five separate batches, two of which were prepared with SPEX Be carrier and three of which were prepared with beryl carrier. The process blanks for these five batches contained $2.1 \cdot 10^{5}$ (average, $\mathrm{n}=2$, SPEX carrier), $3.3 \cdot 10^{5}(\mathrm{n}=1$, SPEX carrier), $4.0 \cdot 10^{3}$ (average, $\mathrm{n}=2$, beryl carrier), $4.7 \cdot 10^{3}\left(\mathrm{n}=1\right.$, beryl carrier), and $5.2 \cdot 10^{3}(\mathrm{n}$ $=1$, beryl carrier) ${ }^{10} \mathrm{Be}$ atoms, respectively. The blank ${ }^{10} \mathrm{Be} /{ }^{9} \mathrm{Be}$ ratios were $1.2 \pm 0.2 \cdot 10^{-14}$ (average, 1SD, $\mathrm{n}=2), 2.0 \pm 0.7 \cdot 10^{-14}(1 \sigma, \mathrm{n}=1), 2.4 \pm 1.2 \cdot 10^{-16}$ (average, $\left.1 \mathrm{SD}, \mathrm{n}=2\right), 2.9 \pm 1.1$ $\cdot 10^{-16}(1 \sigma, \mathrm{n}=1)$, and $3.2 \pm 1.1 \cdot 10^{-16}(1 \sigma, \mathrm{n}=1)$. For the first two batches, processed with commercial Be carrier, we applied a long-term average blank correction equal to a ratio of $1.2 \pm$ $0.2 \cdot 10^{-14}$ ( $1 \mathrm{SD}, \mathrm{n}=19$ batches). For the remaining three batches, processed with beryl carrier, we applied the average blank for the three batches, equal to a ratio of $2.71 \pm 0.76 \cdot 10^{-16}(1 \mathrm{SD}, \mathrm{n}=$ 4). For samples prepared with the commercial carrier, blanks amounted to 1 to $24 \%$ of the total sample ratios. For samples prepared with the beryl carrier, blanks amounted to $<1 \%$ of the total sample ratios.

For Al analysis, samples were processed in five separate batches. The process blanks for these five batches contained $6.9 \cdot 10^{4}(n=1), 6.9 \cdot 10^{4}(n=1), 2.7 \cdot 10^{4}($ average, $n=2), 7.8 \cdot 10^{4}(n=$ 
1), and $2.9 \cdot 10^{4}(\mathrm{n}=1){ }^{26} \mathrm{Al}$ atoms, respectively. The blank ${ }^{26} \mathrm{Al} /{ }^{27} \mathrm{Al}$ ratios were $1.6 \cdot 10^{-15}(\mathrm{n}=1)$, $1.5 \cdot 10^{-15}(\mathrm{n}=1), 6.0 \cdot 10^{-16}($ average, $\mathrm{n}=2), 1.8 \cdot 10^{-15}(\mathrm{n}=1)$, and 6.6 $10^{-16}(\mathrm{n}=1)$. Blanks amounted to $<0.5 \%$ of the total sample ratios. Blank corrections were performed with the average blank for the five batches (equal to a ratio of $1.1 \pm 0.6 \cdot 10^{-15}, 1 \mathrm{SD}$ ).

\section{Retreat rate simulations}

To estimate the rate of latest Pleistocene ice sheet retreat along the sample transect, we considered only the youngest, inheritance-free boulder samples. We systematically simulated potential linear retreat patterns based on the location and uncertainty of each age measurement. The six youngest ${ }^{10} \mathrm{Be}$ measurements and their distances from the present margin were fit to the curve $\mathrm{Y}=\mathrm{B}_{0}+\mathrm{B}_{1} \bullet \mathrm{X} / \mathrm{T}$, where $\mathrm{Y}$ is ${ }^{10} \mathrm{Be}$ age (years), $\mathrm{X}$ is distance from the present margin $(\mathrm{km}), \mathrm{T}$ is the transect length $(\mathrm{km}), \mathrm{B}_{0}$ is the time at which the present margin was reached during ice sheet retreat (years), and $\mathrm{B}_{1}$ is the total duration of ice sheet retreat along the transect (years). For each total duration of ice sheet retreat $\left(B_{1}\right)$ between 0 and 3000 years, $B_{0}$ was varied between values of 8500 and 12000 years. The probability that each set of parameters fits the data was calculated from the normal distributions of error in the ${ }^{10} \mathrm{Be}$ measurements, resulting in a twodimensional normal distribution over $\mathrm{B}_{0}-\mathrm{B}_{1}$ space. By summing the probabilities for each value of $\mathrm{B}_{1}$, this distribution can be collapsed into a normal distribution of $\mathrm{B}_{1}$ values. The maximum of this distribution is the statistically most likely retreat rate. 
TABLE 1. ISOTOPIC INFORMATION FOR SAMPLES FROM UPERNAVIK, NW GREENLAND

\begin{tabular}{|c|c|c|c|c|c|c|c|}
\hline Sample Name & Quartz Mass (g) & $\begin{array}{c}\text { Total Be } \\
\text { Spike }(\mu g)^{*}\end{array}$ & ${ }^{10} \mathrm{Be} /{ }^{\beta} \mathrm{Be}$ Ratio $^{\dagger}$ & $\begin{array}{l}{ }^{10} \mathrm{Be} /{ }^{6} \mathrm{Be} \text { Ratio } \\
\text { Uncertainty }\end{array}$ & Total Al $(\mu \mathrm{g})^{* *}$ & ${ }^{26} \mathrm{Al} /{ }^{27} \mathrm{Al} \mathrm{Ratio}{ }^{\dagger}$ & $\begin{array}{r}{ }^{26} \mathrm{Al}^{27} \mathrm{Al} \text { Ratio } \\
\text { Uncertainty }^{8}\end{array}$ \\
\hline GU091 & 21.76 & 251 & $7.81 \times 10^{-14}$ & $3.08 \times 10^{-15}$ & $\ldots$ & $-{ }_{-}$ & - \\
\hline GU092 & 17.41 & 252 & $5.15 \times 10^{-14}$ & $2.76 \times 10^{-15}$ & $\ldots$ & $\ldots$ & $\ldots$ \\
\hline GU093 & 22.85 & 248 & $1.00 \times 10^{-13}$ & $2.99 \times 10^{-15}$ & $\ldots$ & $\ldots$ & $-\ldots$ \\
\hline GU094 & 11.57 & 247 & $3.60 \times 10^{-14}$ & $1.30 \times 10^{-15}$ & $\ldots$ & $\ldots$ & $\ldots$ \\
\hline GU095 & 22.11 & 247 & $8.85 \times 10^{-14}$ & $2.03 \times 10^{-15}$ & - & $\ldots$ & $\ldots$ \\
\hline GU096 & 16.87 & 248 & $9.23 \times 10^{-13}$ & $2.09 \times 10^{-14}$ & 2429 & $1.62 \times 10^{-12}$ & $3.89 \times 10^{-14}$ \\
\hline GU097 & 20.49 & 248 & $2.18 \times 10^{-13}$ & $4.95 \times 10^{-15}$ & 3858 & $2.82 \times 10^{-13}$ & $1.48 \times 10^{-14}$ \\
\hline GU098 & 20.45 & 248 & $1.03 \times 10^{-13}$ & $2.35 \times 10^{-15}$ & 3257 & $1.65 \times 10^{-13}$ & $1.45 \times 10^{-14}$ \\
\hline GU099 & 20.09 & 248 & $8.58 \times 10^{-14}$ & $1.95 \times 10^{-15}$ & $\ldots$ & $\ldots$ & $\ldots$ \\
\hline GU100 & 20.83 & 246 & $1.28 \times 10^{-13}$ & $2.92 \times 10^{-15}$ & 3699 & $1.82 \times 10^{-13}$ & $8.17 \times 10^{-15}$ \\
\hline GU101 & 8.75 & 247 & $2.63 \times 10^{-14}$ & $1.10 \times 10^{-15}$ & $\ldots$ & $\ldots$ & $\ldots$ \\
\hline GU102 & 14.85 & 253 & $4.18 \times 10^{-13}$ & $1.26 \times 10^{-14}$ & 2493 & $7.20 \times 10^{-13}$ & $6.99 \times 10^{-14}$ \\
\hline GU103 & 16.69 & 252 & $8.84 \times 10^{-13}$ & $2.47 \times 10^{-14}$ & 2229 & $1.73 \times 10^{-12}$ & $5.14 \times 10^{-14}$ \\
\hline GU106 & 18.81 & 248 & $2.58 \times 10^{-13}$ & $5.86 \times 10^{-15}$ & 3512 & $3.52 \times 10^{-13}$ & $1.22 \times 10^{-14}$ \\
\hline GU107 & 5.43 & 248 & $3.18 \times 10^{-14}$ & $1.37 \times 10^{-15}$ & $\ldots$ & $\ldots$ & $\ldots$ \\
\hline GU104 & 21.03 & 248 & $7.63 \times 10^{-14}$ & $1.48 \times 10^{-15}$ & 3085 & $1.31 \times 10^{-13}$ & $1.67 \times 10^{-14}$ \\
\hline GU105 & 23.25 & 247 & $3.32 \times 10^{-13}$ & $7.56 \times 10^{-15}$ & 3484 & $4.48 \times 10^{-13}$ & $1.50 \times 10^{-14}$ \\
\hline GU108 & 17.81 & 246 & $8.04 \times 10^{-14}$ & $2.83 \times 10^{-15}$ & $\ldots$ & $\ldots$ & $\ldots$ \\
\hline GU109 & 17.61 & 258 & $5.88 \times 10^{-14}$ & $2.85 \times 10^{-15}$ & $\ldots$ & $\ldots$ & $\ldots$ \\
\hline GU110 & 17.86 & 248 & $6.10 \times 10^{-13}$ & $1.60 \times 10^{-14}$ & 11120 & $1.91 \times 10^{-13}$ & $8.37 \times 10^{-15}$ \\
\hline GU111 & 15.65 & 244 & $4.09 \times 10^{-13}$ & $9.31 \times 10^{-15}$ & 3289 & $5.04 \times 10^{-13}$ & $1.63 \times 10^{-14}$ \\
\hline GU112 & 16.25 & 248 & $1.18 \times 10^{-13}$ & $2.70 \times 10^{-15}$ & 2764 & $2.03 \times 10^{-13}$ & $9.85 \times 10^{-15}$ \\
\hline GU113 & 21.51 & 248 & $3.08 \times 10^{-13}$ & $7.04 \times 10^{-15}$ & 4515 & $3.23 \times 10^{-13}$ & $1.16 \times 10^{-14}$ \\
\hline GU114 & 14.60 & 247 & $4.90 \times 10^{-14}$ & $1.46 \times 10^{-15}$ & $\ldots$ & $\ldots$ & $\ldots$ \\
\hline GU001 & 21.16 & 248 & $1.31 \times 10^{-13}$ & $3.09 \times 10^{-15}$ & 3854 & $1.66 \times 10^{-13}$ & $7.97 \times 10^{-15}$ \\
\hline GU002 & 21.28 & 248 & $1.03 \times 10^{-13}$ & $2.44 \times 10^{-15}$ & 4214 & $1.25 \times 10^{-13}$ & $9.66 \times 10^{-15}$ \\
\hline GU006 & 11.09 & 248 & $7.86 \times 10^{-14}$ & $1.87 \times 10^{-15}$ & $\ldots$ & 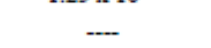 & 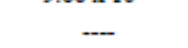 \\
\hline GU017 & 8.14 & 248 & $4.55 \times 10^{-14}$ & $1.40 \times 10^{-15}$ & $\ldots$ & $\ldots$ & $\ldots$ \\
\hline GU041 & 21.42 & 245 & $1.02 \times 10^{-12}$ & $2.41 \times 10^{-14}$ & 2749 & $1.53 \times 10^{-12}$ & $4.06 \times 10^{-14}$ \\
\hline GU042 & 21.04 & 249 & $6.34 \times 10^{-13}$ & $1.49 \times 10^{-14}$ & 3023 & $9.29 \times 10^{-13}$ & $2.91 \times 10^{-14}$ \\
\hline GU043 & 20.02 & 248 & $5.96 \times 10^{-13}$ & $1.47 \times 10^{-14}$ & 3535 & $7.92 \times 10^{-13}$ & $2.27 \times 10^{-14}$ \\
\hline GU044 & 20.48 & 248 & $7.14 \times 10^{-13}$ & $1.62 \times 10^{-14}$ & 3759 & $8.66 \times 10^{-13}$ & $2.77 \times 10^{-14}$ \\
\hline GU045 & 19.33 & 248 & $4.45 \times 10^{-13}$ & $1.01 \times 10^{-14}$ & 3793 & $4.83 \times 10^{-13}$ & $1.71 \times 10^{-14}$ \\
\hline
\end{tabular}

*Refers to the Be spike added through carrier.

${ }^{\dagger}$ Ratios were measured at Lawrence Livermore National Laboratory and have already been blank corrected.

'Reported uncertainties are internal AMS uncertainties.

**Refers to the cumulative quantified $\mathrm{Al}$, including both $\mathrm{Al}$ spike added through carrier and native $\mathrm{Al}$ in the quartz. 